\title{
Calculate Accurate 3D Cost Distance Efficiently
}

\author{
Yaqian Chen a , Jiangfeng She ${ }^{\text {a }}$, Xingong Li ${ }^{\text {b, } *}$ \\ a School of Geography and Ocean Science, Jiangsu Provincial Key Laboratory of Geographic Information Science and Technology, \\ Nanjing University, Nanjing, China, chenyaqian0202@163.com, gisjf@nju.edu.cn \\ ${ }^{b}$ Department of Geography \& Atmospheric Science, University of Kansas, Lawrence, KS 66045 USA, lixi@ku.edu \\ * Corresponding author
}

Keywords: Voxel, Dijkstra Algorithm, Binary Heap, Route Planning

\begin{abstract}
:
Cost distance is one of the fundamental functions in geographic information systems (GIS), which has been used in various applications such as route planning, construction of Thiessen polygons and distance weighted interpolation. Conventional 2D cost distance function, due to its limited movement directions (either 4 or 8 neighbourhood cells) in the raster data model, overestimates the least cost and the problem is especially severe with a homogeneous friction surface. 3D cost distance function removes the limitation that movement must occur on a planar surface. It can therefore take into account tunnels and bridges when calculating least cost paths. In addition, it can also be used in many other application domains which deal with 3D geospatial data such as in atmospheric science, geology, and oceanography. Based on the method in Tomlin (2010), which can completely eliminate the overestimation when traveling on a homogenous friction surface, this research proposes an algorithm that calculates accurate least cost with both homogeneous and heterogeneous friction in $3 \mathrm{D}$ space. When extending the cost distance function from $2 \mathrm{D}$ to $3 \mathrm{D}$, the number of voxels in the propagation front increases significantly and efficiency is an imperative issue. This research also improves the computational efficiency by developing a data structure that combines a binary heap and a hash table. Our results show that the proposed algorithm can calculate accurate $3 \mathrm{D}$ cost distance in a homogeneous friction space, and the proposed data structure (i.e., heap plus hash table) not only significantly reduces the algorithm's runtime but also benefits more in $3 \mathrm{D}$ than in $2 \mathrm{D}$. In addition, we have applied the method in a 3D drone delivery routing application in a city environment (Figure 1). Additional applications, such as calculating groundwater flow paths of least hydraulic resistance in a heterogeneous 3D hydraulic conductivity field, are currently under development.
\end{abstract}

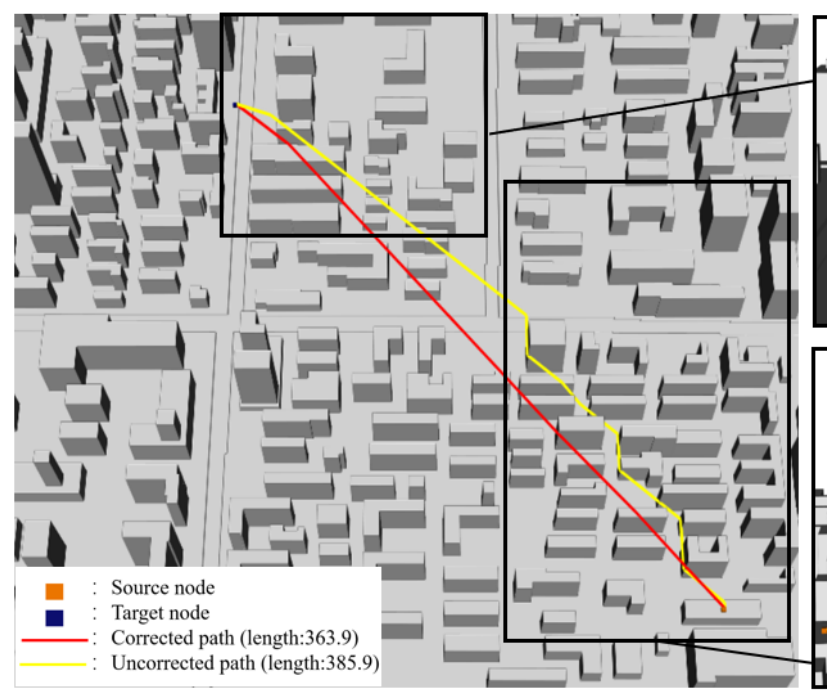

Top view

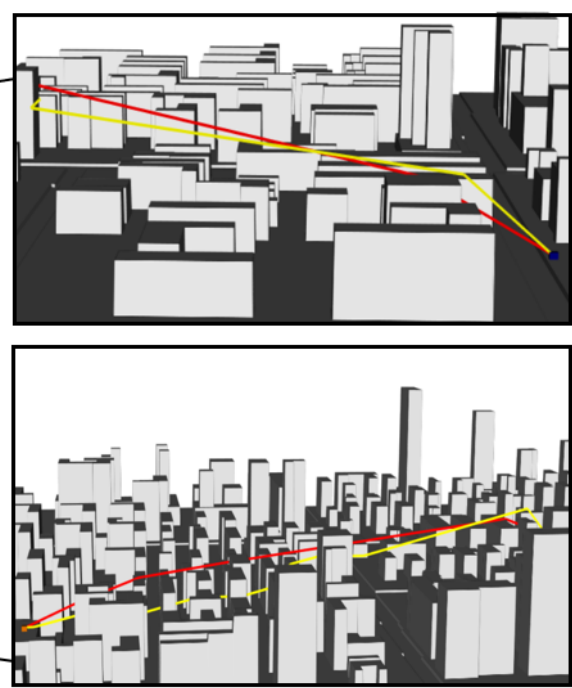

Side view

Figure 1.3D shortest drone delivery path in a city environment with a top view and two close-up side views on the route. 